\title{
Influencia de la edad sobre el cumplimiento de las recomendaciones de actividad física: Resultados de la Encuesta Nacional de Salud en Chile 2009-2010
}

\author{
Adherence to physical activity recommendations across age categories: \\ Findings from the 2009-2010 Chilean National Health Survey
}

\section{RESUMEN}

A pesar de que la actividad física (AF) es un factor protector contra las enfermedades crónicas no transmisibles, un gran porcentaje de la población no cumple los niveles mínimos recomendados. El objetivo fue investigar como varían los niveles de práctica de $A F$ entre los diferentes grupos etarios en la población chilena. Se incluyeron 5.133 participantes de la Encuesta Nacional de Salud 2009-2010. La AF fue determinada utilizando el cuestionario GPAQ. La inactividad física fue definida como $<600 \mathrm{MET} / \mathrm{min} /$ semana de AF moderada a vigorosa. La asociación entre AF y edad fue investigada por sexo mediante regresión logística. Las mujeres presentaron una mayor probabilidad de ser físicamente inactivas en comparación a los hombres $(p<0,0001)$. Al analizar la prevalencia de inactividad física por sexo y grupo etario, esta cambió ligeramente entre los 20 a 59 años, pero a partir de los 60 se observó un incremento importante en la prevalencia llegando a $63 \%$ y $56 \%$ para mujeres y hombres $>80$ años, respectivamente. Los resultados obtenidos confirman la necesidad de seguir fomentando la práctica regular de AF física a través de todo el ciclo vital, pero en especial sobre los 60 años, ya que este grupo presenta una mayor probabilidad de ser físicamente inactivo.

Palabras clave: Actividad física; Edad; Ejercicio; Envejecimiento; Factores de riesgo.

\footnotetext{
ABSTRACT

Although physical activity $(P A)$ is a protective factor against non-communicable diseases, there is an important proportion of the population who do not meet PA guidelines. Our aim was to investigate how adherence to $P A$ guidelines varies across different age groups in the Chilean population. 5,133 participants from the 2009-2010 Chilean Health Survey were included in this study. PA was measured using the Global Physical Activity Questionnaire (GPAQ). Physical inactivity was defined as $<600 \mathrm{MET} / \mathrm{min} /$ week of moderate to vigorous intensity $P$ A. Logistic regression was used to investigate the association of $P A$ with age. The main finding of this shows that women were more likely to be inactive than men $(p<0.0001)$. Physical inactivity prevalence varied
}

Alex Garrido-Méndez ${ }^{1,14^{*}}$, Yeny Concha-Cisternas ${ }^{2,3^{*}}$, Fanny Petermann Rocha ${ }^{4^{*}}$, Ximena Díaz-Martínez ${ }^{5}$, Ana María Leiva ${ }^{6}$, Claudia Troncoso ${ }^{7}$, María Adela Martinez ${ }^{8}$, Carlos Salas-Bravo ${ }^{9}$, Cristian Álvarez ${ }^{10}$, Rodrigo Ramírez-Campillo ${ }^{10}$, Carlos Cristi-Montero ${ }^{11}$, Fernando Rodríguez ${ }^{11}$, José A Iturra-González ${ }^{12}$, Carlos Celis-Morales ${ }^{4,13}$, en representación de los integrantes del grupo ELHOC (Epidemiology of Lifestyle and Health Outcomes in Chile).

${ }^{*} A G M, Y C, F P R$ contribuyeron de igual manera a este manuscrito por ende son considerados como primer autor compartido.

1. Departamento de Ciencias del Deporte y Acondicionamiento Físico, Universidad Católica de la Santísima Concepción, Concepción, Chile. 2. Escuela de Kinesiología, Facultad de Salud, Universidad Santo Tomás, Chile.

3. Universidad Tecnológica de Chile INACAP, Chile. 4. BHF Glasgow Cardiovascular Research Centre, Institute of Cardiovascular and Medical Sciences, University of Glasgow, Glasgow, United Kingdom

5. Grupo de Investigación Calidad de Vida, Departamento Ciencias de la Educación, Universidad del Biobío, Chillán, Chile. 6. Instituto de Anatomía, Facultad de Medicina, Universidad Austral de Chile, Valdivia, Chile. 7. Facultad de Medicina. Departamento de Ciencias Clínicas y Preclínicas.

Universidad Católica de la Santísima Concepción, Chile. 8. Instituto de Farmacia, Facultad de Ciencias, Universidad Austral de Chile, Valdivia, Chile.

9. Departamento de Educación Física, Facultad de Educación, Universidad de Concepción, Concepción, Chile.

10. Departamento de Ciencias de la Actividad Física Núcleo de Investigación en Salud, Actividad Física y Deporte, Universidad de los Lagos, Osorno, Chile. 11. Grupo IRyS, Escuela de Educación Física,

Pontificia Universidad Católica de Valparaíso, Valparaíso, Chile. 12. Escuela Kinesiología en Universidad Católica Cardenal Raúl Silva Henríquez, Santiago, Chile.

3. Centro de Investigación en Fisiología del Ejercicio - CIFE, Universidad Mayor. Santiago, Chile

14. Escuela de Educación Física, Universidad San Sebastián, Concepción, Chile.

Dirigir correspondencia a: Dr. Carlos Celis-Morales. BHF Glasgow Cardiovascular Research Centre. 126 University Avenue. Glasgow University. Glasgow, United Kingdom, G12 8TA. Teléfono: +(0)4401413304201 Email: carlos.celis@glasgow.ac.uk

Este trabajo fue recibido el 30 de enero de 2018. Aceptado con modificaciones: 12 de julio de 2018. Aceptado para ser publicado: 18 de octubre de 2018.

slightly between 20 and 59 years. However, among those individuals aged $>60$ years the prevalence increased markedly, reaching $63 \%$ and $56 \%$ for women and men aged 
$>80$ years, respectively. These findings confirm the necessity of promoting PA across the lifespan, but more importantly, these results suggest that more promotion and opportunities for engaging in PA should be created especially for older adults, who are more likely to be physically inactive.

Keywords: Age; Ageing; Exercise; Physical activity; Risk factors.

\section{INTRODUCCIÓN}

A nivel mundial la escasa práctica de actividad física (AF) es uno de los principales factores de riesgo que genera efectos nocivos sobre la salud $d^{1,2,3}$. Actualmente, la inactividad física, es decir, realizar menos de 150 minutos de AF de intensidad moderada por semana ${ }^{4}$, es el cuarto factor de riesgo de mortalidad más importante, atribuyéndole el $6 \%$ de las defunciones anuales en todo el mundo ${ }^{5}$. Estudios también han demostrado que la inactividad física es un importante factor de riesgo para el desarrollo de enfermedades crónicas no transmisibles (ECNT), como la diabetes mellitus tipo 2 (DMT2), hipertensión arterial (HTA), obesidad y algunos tipos de cáncer ${ }^{3,6,7,7,9,10,11,12,13,14,15}$. Por otra parte, la inactividad física se ha asociado con deterioro físico y cognitivo, lo que la vincula a fragilidad y mayor riesgo de dependencia ${ }^{15,16,17,18,19,20}$.

A pesar de la abundante evidencia científica que demuestra los beneficios de realizar $\mathrm{AF}$, un gran porcentaje de la población no cumple con los niveles mínimos recomendados para mantener un óptimo estado de salud ${ }^{21,22}$. Reportes demuestran que actualmente un 31,1\% de la población adulta no cumple con las recomendaciones mínimas de $\mathrm{AF}^{23}$, mientras que, en Chile, según datos de la Encuesta Nacional de Salud 2009-2010 (ENS 2009-2010), un 27,1\% de la población reportó ser físicamente inactiva ${ }^{24}$.

Si bien estos hallazgos han generado alerta, propiciando la creación de políticas públicas orientadas a la reducción de la inactividad física en todos los grupos etarios, los esfuerzos aún continúan siendo insuficientes para incentivar y promover la realización de AF en todas las edades, siendo aún más difícil promoverla en adultos mayores ${ }^{25}$.

Considerando los grandes beneficios que otorga la AF a la salud, la autonomía y la prevención de enfermedades durante todo el ciclo de la vida $3,6,7,8,9,10,11,12,13,14$, pero por sobre todo durante la etapa de envejecimiento ${ }^{5}$, es fundamental identificar cómo la práctica de AF varía en los diferentes rangos etarios, logrando, de esta manera, orientar y generar intervenciones hacía los grupos más vulnerables. Por lo anterior, el objetivo del presente estudio fue investigar como varían los niveles de práctica de AF entre los diferentes grupos etarios en la población chilena.

\section{MATERIAL Y METODOS Diseño muestral}

La muestra seleccionada incluyó a los participantes ENS, desarrollada en Chile entre octubre del año 2009 y septiembre del año 201024. La ENS 2009-2010 corresponde a un estudio poblacional de corte transversal realizado en hogares, con una muestra nacional, probabilística, estratificada y multietápica de 5.412 personas mayores de 15 años con representatividad nacional, regional, y área urbana/rural. Un total de 5.133 participantes con información disponible en relación con su nivel de AF fueron incluidos en este estudio. El protocolo del estudio fue aprobado por el Comité de Ética de Investigación de la Escuela de Medicina de la Pontificia Universidad Católica de Chile, y todos los participantes firmaron voluntariamente un consentimiento informado ${ }^{24}$.

\section{Mediciones antropométricas y de estilo de vida}

El estado nutricional fue clasificado en base a los puntos de corte de la Organización Mundial de la Salud (OMS): Bajo peso: $<18,5 \mathrm{~kg} / \mathrm{m}^{2}$; Normopeso: $18,5-24,9 \mathrm{~kg} / \mathrm{m}^{2}$; Sobrepeso: $25,0-29,9 \mathrm{~kg} / \mathrm{m}^{2}$ y Obesidad: $\geq 30,0 \mathrm{~kg} / \mathrm{m}^{2(26)}$, mientras que la obesidad central fue definida como un perímetro de cintura $(P C) \geq 83 \mathrm{~cm}$ para mujeres $y \geq 88 \mathrm{~cm}$ para hombres, según los puntos de corte sugeridos por la ENS en población chilena $^{24}$. Las variables sociodemográficas (edad, sexo, nivel educacional, ingreso económico, zona geográfica) y datos asociados con el estilo de vida, como el tabaquismo, auto-reporte de salud y bienestar, comorbilidad (DMT2, HTA y síndrome metabólico) y consumo de alcohol, frutas y verduras se obtuvieron mediante la aplicación de cuestionarios validados en la ENS 2009-2010.

\section{Clasificación de actividad física}

El tiempo destinado para la AF relacionada al transporte (ej. caminar, andar en bicicleta) y actividades de intensidad moderada o vigorosa realizadas durante el tiempo libre y/o en el trabajo, fueron determinados según la guía de análisis de GPAQ (Global Physical Activity Questionnaire v2 $)^{27}$. Para determinar los niveles de AF total, las variables fueron expresadas en METs (Metabolic energy equivalents). Se consideró como punto de corte para inactividad física un gasto energético <600 METs/minutos/semana, o su equivalente de 150 minutos de $\mathrm{AF}$ de intensidad moderada a vigorosa o 75 minutos de AF de intensidad vigorosa a la semana o su combinación, según las recomendaciones de la OMS y especificaciones de la guía de análisis de GPAQ $5^{27}$. Los niveles de sedentarismo fueron determinados mediante el auto-reporte de tiempo destinado a actividades que involucren estar sentado o reclinado durante el tiempo libre o de trabajo (ej. tiempo sentado frente al computador, viendo televisión, viajando en bus, tren o auto, etc.).

\section{Análisis estadísticos}

Las diferencias entre niveles de $\mathrm{AF}$ (activo versus inactivo) fueron determinadas con t-test para variables continuas con distribución normal y el test de Mann-Whitney para variables que no cumplían con una distribución normal. El test Chi-cuadrado fue utilizado para variables categóricas.

Para investigar la asociación entre edad y AF se utilizó análisis de regresión logística no lineal, donde la edad fue introducida en el modelo estadístico como variable continua, estableciendo como grupo de referencia participantes con 
una edad de 46 años, que representa la mediana de la población de la ENS 2009-2010. La AF fue codificada en dos categorías (físicamente activo $=0$ y físicamente inactivo $=$ 1). Los datos para estos análisis fueron presentados como Odds ratio (OR) y sus respectivos intervalos de confianza de $95 \%(95 \%$ IC). Todos los análisis fueron ajustados por nivel educacional, zona de residencia (urbano/rural), ingreso económico, índice de masa corporal (IMC), tabaquismo, consumo de frutas y verduras, consumo de alcohol, autoreporte de estado de salud y bienestar y comorbilidad (DMT2, HTA y síndrome metabólico).

Para todos los análisis se utilizó el módulo de muestras complejas del programa STATA SE v14, y todos los resultados fueron estimados utilizando muestras expandidas según la ENS 2009-2010²4. El nivel de significancia fue definido como $\mathrm{p}<0,05$.

\section{RESULTADOS}

Las características de la población estudiada, según niveles de AF, son presentadas en la tabla 1. Se evidenció que los participantes clasificados como físicamente inactivos fueron principalmente mujeres $(66,9 \%)$, presentaron mayor edad y menor nivel educacional. A nivel antropométrico, las personas físicamente inactivas poseían una mayor prevalencia de obesidad y obesidad central. Con respecto al estilo de vida, las personas físicamente inactivas mostraron hábito tabáquico y mayor tiempo destinado al desarrollo de actividades de tipo sedente.

En la figura 1 se presenta la prevalencia de inactividad física según grupo etario y sexo. Estos resultados señalan que la prevalencia de inactividad física es similar a edades tempranas, entre 20 a 60 años para mujeres y hombres; sin embargo, después de los 60 años la prevalencia aumentó rápidamente para ambos sexos, llegando a $63 \%$ en mujeres y $56 \%$ en hombres con una edad mayor a 80 años en comparación a $25 \%$ y $9 \%$ en mujeres y hombres con edades menor a 20 años, respectivamente.

Al investigar la probabilidad de ser físicamente inactivo, esta fue mayor en mujeres que en hombres (OR: 1,51 [95\% IC: 1,32; 1,72], p<0,0001). Cuando se investigó la asociación entre inactividad física y edad, tomando como referencia los participantes con una edad de 46 años, que representa la mediana de la población incluida en este estudio, se observó que para los hombres existió una probabilidad de ser físicamente inactivo en todas las edades menores a 55 años; no obstante, la probabilidad de no cumplir con las recomendaciones de $\mathrm{AF}$ incrementó significativamente en un 36\% a partir de los 60 años en comparación al grupo de referencia (OR: 1,36 [95\% IC: 1,01; 1,85], $p=$ 0,019 ). En el caso de las mujeres, la probabilidad de ser físicamente inactiva fue similar entre los 30 a 55 años en comparación al grupo de referencia (46 años de edad), pero esta probabilidad incrementó en un 34\% en mujeres con edades menores a 25 años (OR: 1,34 [95\% IC: 1,01; $1,79], p=0,021)$ y en un $32 \%$ a partir de los 60 años (OR: 1,32 [95\% IC: 1,$06 ; 1,65], p=0,003$ ) (Tabla 2 y Figura 2). En comparación al grupo de referencia, la probabilidad de ser físicamente inactivo se incrementó en un $32 \%$, $63 \%, 109 \%, 180 \%$ y $295 \%$ para mujeres con edades de $60,65,70,75$ y >80 años, respectivamente; mientras que para hombres la probabilidad de ser físicamente inactivo incrementó en un $36 \%, 78 \%, 137 \%, 223 \%$ y $370 \%$ para las edades correspondientes a 60, 65, 70, 75 y $>80$ años, respectivamente (Tabla 2 y Figura 2).

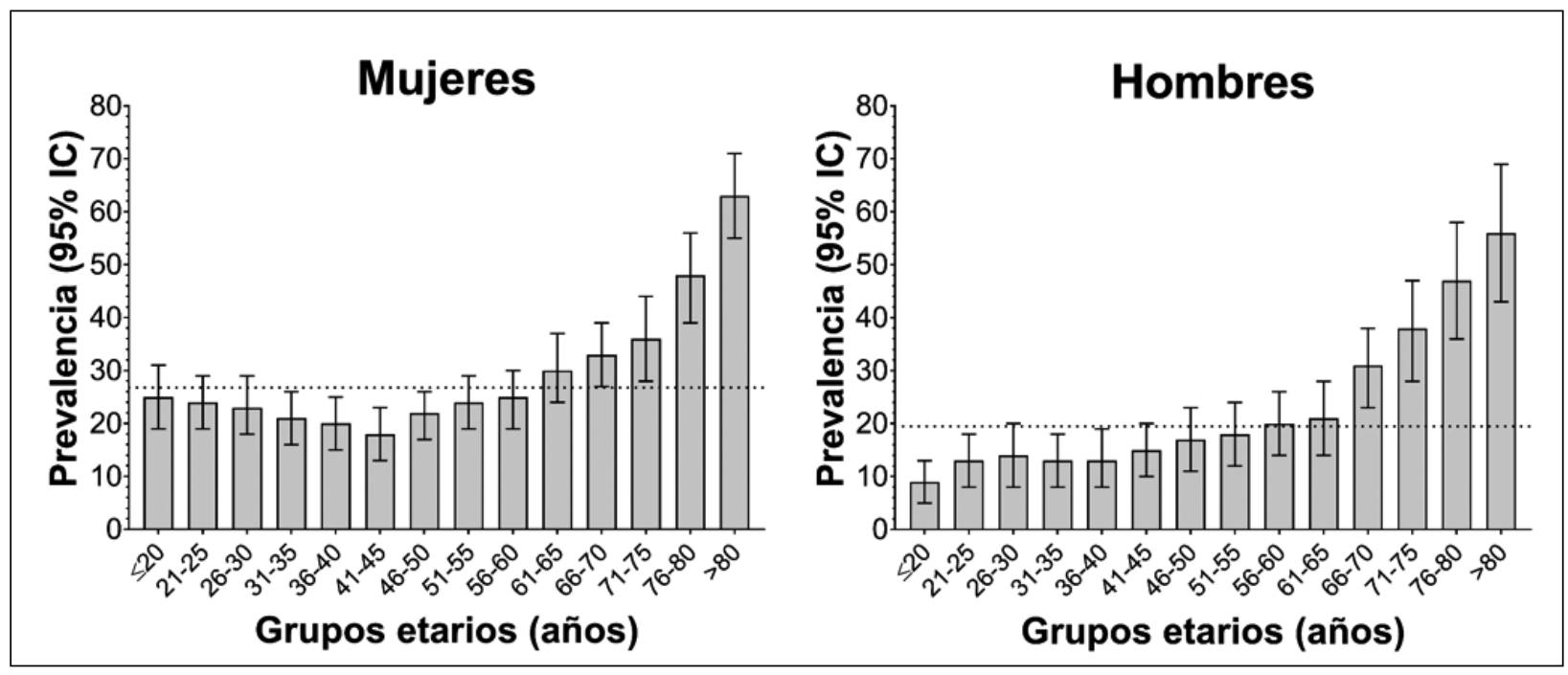

Figura 1. Asociación entre edad y la prevalencia de inactividad física en mujeres y hombres.

Los resultados son presentados como prevalencia y su respectivo 95\% IC. La línea negra punteada indica la prevalencia nacional para mujeres y hombres, respectivamente. 
Tabla 1. Características de la población chilena según nivel de actividad física.

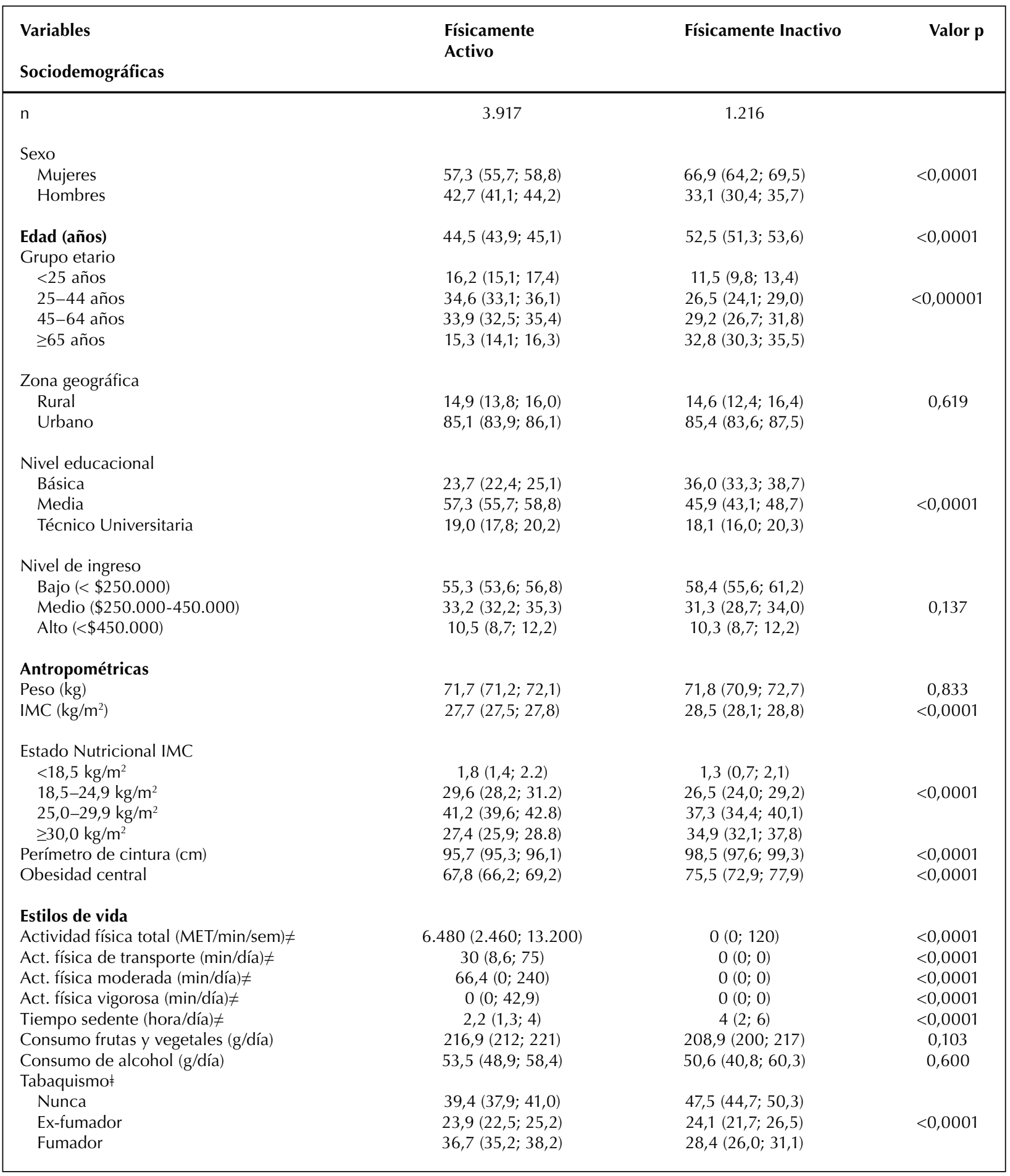

Datos presentados: medias para variables continuas y \% para variables categóricas, ambos con intervalo de confianza del 95\%. Variables sin distribución normal: mediana y rangos inter cuartilf. Diferencias entre variables continuas con distribución normal: t-test; y Mann-Whitney test para variables sin distribución normal. Variables categóricas: Chi-cuadrado. Para inactividad física se utilizó un punto de corte en gasto energético <600 METs/minutos/ semana, según OMS y especificaciones de la guía de análisis de GPAQ. 

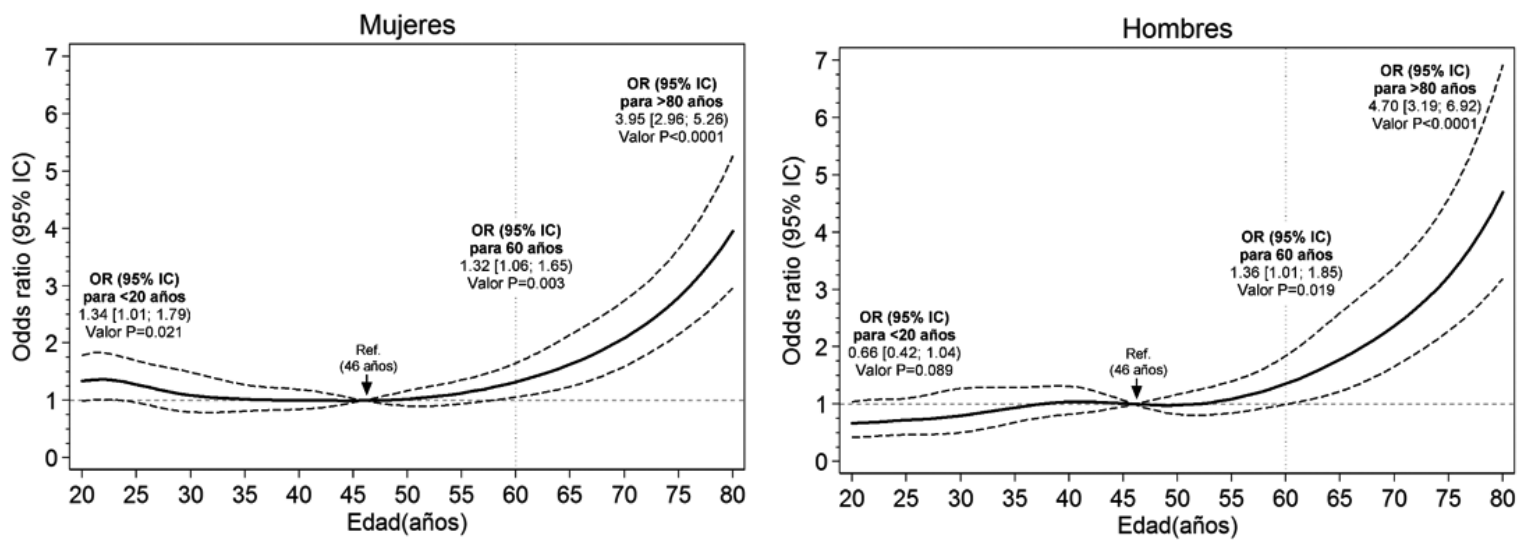

Figura 2. Asociación entre edad y probabilidad de ser físicamente inactivo en mujeres y en hombres.

Datos son presentados como Odds ratio (línea negra continua) y su respectivo 95\% IC (línea negra punteada). Los participantes con una edad de 46 años fueron utilizados como grupo de referencia, ya que este valor representa la mediana tanto para mujeres como para hombres en la ENS. La línea punteada de color gris en posición horizontal indica el valor de no asociación (Odds ratio $=1$ ), por ende, un Odds ratio menor a 1 indica que la población tiene mayor posibilidad de cumplir con la recomendación de AF, mientras que un Odds ratio $>1$ indica que la población tiene una mayor probabilidad de no cumplir con las recomendaciones de AF de al menos 150 minutos a la semana de actividad física de intensidad moderada a vigorosa o su equivalente en METs (>600 METs/minuto/semana). La línea punteada de color gris ubicada a los 60 años de edad representa el punto donde la asociación comienza a ser significativa mayor tanto en mujeres y hombres. También se presenta el Odds ratio para inactividad física en el grupo $<20$ años y para el grupo $>80$ años. Los análisis fueron ajustados por nivel educacional, zona de residencia (urbano/rural), ingreso económico, IMC, tabaquismo, consumo de frutas y verduras, consumo de alcohol, auto-reporte de estado de salud y bienestar, y comorbilidad (DMT2, HTA y síndrome metabólico).

Tabla 2. Odds ratio para inactividad física según grupo etario en Mujeres y Hombres.

\begin{tabular}{|c|c|c|c|c|}
\hline \multirow[b]{2}{*}{ Grupo etario (años) } & \multicolumn{2}{|c|}{ Mujeres } & \multicolumn{2}{|c|}{ Hombres } \\
\hline & OR $(95 \% \mathrm{Cl})$ & Valor $\mathbf{p}$ & OR $(95 \% \mathrm{Cl})$ & Valor $p$ \\
\hline$\leq 20$ & $1,34(1,01 ; 1,79)$ & 0,021 & $0,66(0,42 ; 1,04)$ & 0,089 \\
\hline 25 & $1,28(0,97 ; 1,69)$ & 0,080 & $0,72(0,47 ; 1,10)$ & 0,173 \\
\hline 30 & $1,09(0,80 ; 1,49)$ & 0,124 & $0,80(0,50 ; 1,27)$ & 0,211 \\
\hline 35 & $1,02(0,81 ; 1,28)$ & 0,890 & $0,94(0,68 ; 1,29)$ & 0,358 \\
\hline 40 & $1,00(0,96 ; 1,04)$ & 0,927 & $1,04(0,82 ; 1,32)$ & 0,407 \\
\hline 46 & 1,00 (referencia) & --- & 1,00 (referencia) & \\
\hline 50 & $1,03(0,90 ; 1,17)$ & 0,856 & $0,98(0,82 ; 1,17)$ & 0,948 \\
\hline 55 & $1,13(0,94 ; 1,36)$ & 0,314 & $1,09(0,85 ; 1,40)$ & 0,651 \\
\hline 60 & $1,32(1,06 ; 1,65)$ & 0,003 & $1,36(1,01 ; 1,85)$ & 0,019 \\
\hline 65 & $1,63(1,24 ; 2,14)$ & $<0,0001$ & $1,78(1,23 ; 2,59)$ & $<0,0001$ \\
\hline 70 & $2,09(1,60 ; 2,74)$ & $<0,0001$ & $2,37(1,66 ; 3,38)$ & $<0,0001$ \\
\hline 75 & $2,80(2,15 ; 3,64)$ & $<0,0001$ & $3,23(2,28 ; 4,59)$ & $<0,0001$ \\
\hline 80 y más & $3,95(2,96 ; 5,26)$ & $<0,0001$ & $4,70(3,19 ; 6,92)$ & $<0,0001$ \\
\hline
\end{tabular}

Datos son presentados como Odds ratio y su respectivo $95 \%$ IC. Los participantes con una edad de 46 años fueron utilizados como grupo de referencia, ya que este valor representa la mediana tanto para mujeres como para hombres en la ENS. Un Odds ratio $<1$ indica que la población tiene mayor posibilidad de cumplir con la recomendación de AF, mientras que un Odds ratio $>1$ indica que la población tiene una mayor probabilidad de no cumplir con las recomendaciones de AF de al menos 150 minutos a la semana de actividad física de intensidad moderada a vigorosa o su equivalente en METs ( $>600 \mathrm{METS} /$ minuto/semana). Los análisis fueron ajustados por nivel educacional, zona de residencia (urbano/rural), ingreso económico, IMC, tabaquismo, consumo de frutas y verduras, consumo de alcohol, auto-reporte de estado de salud y bienestar, y comorbilidad (DMT2, HTA y síndrome metabólico). 


\section{DISCUSIÓN}

El principal resultado de este estudio indica que la prevalencia de inactividad física varía con la edad, llegando a $56 \%$ para hombres y $63 \%$ para mujeres mayores a 80 años en comparación a una prevalencia de $9 \%$ y $25 \%$ para hombres y mujeres menores a 20 años, respectivamente. Otro resultado de interés para la generación o fortalecimiento de políticas de salud pública en $\mathrm{AF}$, tiene relación a la cuantificación de la probabilidad de ser físicamente inactivo según grupo etario. Estos resultados revelan que en comparación a personas en el grupo de referencia correspondiente a 46 años (que representa la edad promedio de la población de la ENS para ambos sexos), la probabilidad de ser físicamente inactivo aumenta significativamente a partir de los 60 años para ambos sexos, llegando a ser un 295\% y $370 \%$ mayor en mujeres y hombres mayores a 80 años, respectivamente. Si bien la promoción de la práctica regular de AF debería aplicarse a través de todo el ciclo de vida (desde la niñez a la etapa de adulto mayor), ya que los beneficios asociados a AF son importantes para todos los grupos etarios, estos resultados sugieren que, a partir de los 60 años, una edad donde la mayor parte de la población comienza su etapa de desvinculación laboral, es cuando debería haber un énfasis especial en la creación de políticas públicas y generación de espacios para la práctica regular de AF, la cual está especialmente enfocada a las características del grupo etario, en este caso, programas de AF especializados para el adulto mayor. Esto podría ofrecer grandes beneficios en relación con lograr una mayor interacción social, independencia y, por ende, mayor probabilidad de lograr un envejecimiento exitoso través de la práctica regular de AF.

Los resultados de nuestro estudio son similares a los reportados por estudios internacionales que muestran un aumento en la prevalencia de inactividad física conforme avanza la edad, fluctuando de un 15 a $38 \%$ en adultos jóvenes (15 a 29 años) y de un 28 a $60 \%$ en adultos mayores de 60 años $^{28,29}$. Similarmente, Hamrik et a ${ }^{30}$. señalan que el aumento de la edad incrementa la proporción de hombres y mujeres que no realizan $\mathrm{AF}$, reportando una probabilidad 4 veces mayor de ser físicamente inactivo al alcanzar los 65 años, en comparación con adultos jóvenes (18-39 años). Los antecedentes descritos permitirían señalar a la edad como un potente factor predictivo de inactividad física, además de sindicar edades "críticas" para esta conducta. Este hallazgo sugiere la necesidad de intervenir tempranamente y de forma continua para compensar la disminución de la práctica de AF durante el ciclo vital, pero, especialmente, durante el envejecimiento.

El aumento en el porcentaje de adultos mayores físicamente inactivos podría asociarse principalmente a los cambios biológicos, desarrollo temprano de enfermedades que limitan la práctica de $\mathrm{AF}$, pero también podría estar asociado a cambios sociales como la jubilación y, finalmente, el poco acceso a programas de AF dirigidos y especializados para esta población ${ }^{31}$. Este patrón de inactividad física predominante en población chilena envejecida ocurre también en países desarrollados y subdesarrollados, lo que genera preocupación considerando los efectos adversos asociados a la inactividad física ${ }^{6,22,32}$. En este contexto, es necesario generar más investigación que nos permita identificar los principales factores que favorecen como también aquellos que limitan la práctica de AF en distintas etapas del ciclo de vida, y en especial los factores asociados a AF durante la desvinculación laboral o en la etapa de adulto mayor, de manera de poder diseñar programas de intervención y políticas públicas que sean adecuadas al contexto nacional y en especial a cada uno de los principales grupos etarios.

Por otro lado, este estudio identificó una asociación entre ser clasificado como físicamente inactivo y factores sociodemográficos como género y bajo nivel educaciona ${ }^{33,34}$. Este resultado coincide con el reportado previamente en población brasileña y chilena, los cuáles señalan que mujeres y personas con baja escolaridad presentan menores niveles de $\mathrm{AF}^{33,34}$. No obstante, este es el primer estudio en Chile que investiga la asociación que existe entre la edad y la realización de AF.

Finalmente, es importante recalcar que la práctica de AF debe ser incorporada desde la primera infancia, reforzándose durante todo el ciclo vital. De esta manera se lograría generar hábitos de vida que permanecerán durante la etapa adulta y permitirían aumentar la probabilidad de alcanzar un envejecimiento exitoso. Un estudio publicado recientemente refuerza este punto y entrega evidencia del impacto que tiene sobre la fragilidad en el adulto mayor el tener un bajo nivel de fitness cardio-respiratorio y fuerza muscular durante la adolescencia. En este estudio el riesgo de fragilidad en la tercera edad fue un $89 \%$ mayor en personas que tuvieron un nivel bajo de fuerza muscular y bajo nivel de fitness en su adolescencia en comparación personas con niveles altos ${ }^{35}$.

\section{Fortalezas y limitaciones del estudio}

La principal fortaleza del estudio es que sus resultados son representativos para población chilena, e incluyen un rango de edad amplio que permite estudiar los patrones de AF durante el ciclo vital (15 a 80 años). Además cabe destacar que los datos relacionados a niveles de AF han sido recolectados utilizando instrumentos validados ${ }^{36}$.

Dentro de las limitantes del estudio se encuentra el auto-reporte de AF, el cual presenta una baja correlación con métodos objetivos para la cuantificación de los niveles de AF, como, por ejemplo, la acelerometría ${ }^{36,37}$. Por ende, las asociaciones observadas en este estudio podrían estar sesgadas por la sobreestimación de AF realizada, principalmente en personas con estilos de vida no saludable, con patologías existentes y aquellas con sobrepeso u obesidad $^{36,37}$. Finalmente, es importante considerar que, debido al diseño observacional de corte transversal de la ENS, no se puede establecer una relación de causa-efecto.

\section{CONCLUSIONES}

Los hallazgos de este estudio permiten señalar que existen 
edades críticas para hombres y mujeres, principalmente sobre los 60 años, en donde aumenta de forma significativa la probabilidad de no cumplir con las recomendaciones de AF en comparación a población más joven (20-59 años). Estos resultados son relevantes, ya que aportan información y evidencia que puede permitir la creación de políticas gubernamentales que incentiven la promoción y la generación de espacios y oportunidades para la práctica de AF en los distintos grupos etarios, pero en especial en aquellos que, debido a cambios sociales, laborales, como lo son la desvinculación laborar, o por problemas relacionados a su salud, reducen sus niveles de AF considerablemente a partir de los 60 años. Para aumentar la adherencia a la práctica regular de AF, la promoción y creación de espacios y programas debe comenzar en una edad temprana y no solo en la tercera edad. Pero también se debe considerar que la promoción y creación de oportunidades y espacios para la práctica de AF deber ser adecuada y personalizada según el grupo etario al que deseamos intervenir, lo cual no solo aumentaría la adherencia, sino también los beneficios asociados a una práctica regular de AF.

Agradecimientos. Se agradece de manera especial a todos los participantes de la ENS 2009-2010, al equipo profesional de la Escuela de Salud Pública, de la Facultad de Medicina de la Pontificia Universidad Católica de Chile, quienes desarrollaron y aplicaron la Encuesta Nacional de Salud y al Ministerio de Salud del Gobierno de Chile. Todos los autores revisaron críticamente el manuscrito y están de acuerdo con su versión final. Ninguno de los autores reporta tener conflictos de intereses.

\section{BIBLIOGRAFIA}

1. Celis-Morales CA, Lyall DM, Welsh P, Anderson I, Steell L, Guo $Y$, et al. Association between active commuting and incident cardiovascular disease, cancer, and mortality: prospective cohort study. BMJ 2017; 357(4): j1456.

2. Yates T, Zaccardi F, Dhalwani NN, Davies MJ, Bakrania K, Celis-Morales CA, et al. Association of walking pace and handgrip strength with all-cause, cardiovascular, and cancer mortality: a UK Biobank observational study. Eur Heart I 2017; 1(1): ehx449.

3. Celis-Morales CA, Lyall DM, Anderson J, Iliodromiti S, Fan $Y$, Ntuk UE, et al. The association between physical activity and risk of mortality is modulated by grip strength and cardiorespiratory fitness: evidence from 498135 UK-Biobank participants. Eur Heart J 2017; 38(2): 116-122.

4. Tremblay MS, Aubert S, Barnes ID, Saunders TJ, Carson $V$, Latimer-Cheung $A E$, et al. Sedentary Behavior Research Network (SBRN) - Terminology Consensus Project process and outcome. Int J Behav Nutr Phys Act. 2017; 14.

5. World Health Organization. Global recommendations on physical activity for health. 2010. http://www.who.int/ dietphysicalactivity/factsheet_recommendations/en/.

6. Celis-Morales C, Salas C, Alduhishy A, Sanzana R, Martinez $M$, Leiva $A$, et al. Socio-demographic patterns of physical activity and sedentary behaviour in Chile: results from the National Health Survey 2009-2010. J Public Health 2015; 38(2): e98-e105.
7. Celis-Morales C, Salas C, Alvarez C, Farias NA, Campillos RR, Leppe J, et al. Higher physical activity levels are associated with lower prevalence of cardiovascular risk factors in Chile. Rev Med Chil 2015; 143(11): 1435-1443.

8. Celis-Morales CA, Perez-Bravo F, Ibanes L, Sanzana $R$, Hormazabal E, Ulloa N, et al. Insulin Resistance in Chileans of European and Indigenous Descent: Evidence for an Ethnicity x Environment Interaction. PlosOne 2011; 6(9): e24690.

9. Celis-Morales CA, Petermann F, Hui L, Lyall DM, Iliodromiti S, McLaren J, et al. Associations Between Diabetes and Both Cardiovascular Disease and All-Cause Mortality Are Modified by Grip Strength: Evidence From UK Biobank, a Prospective Population-Based Cohort Study. Diabetes Care 2017; 40(12): 1710-1718.

10. Yates T, Zaccardi F, Dhalwani NN, Davies MJ, Bakrania K, Celis-Morales CA, et al. Association of walking pace and handgrip strength with all-cause, cardiovascular, and cancer mortality: a UK Biobank observational study. Eur Heart I 2017; 38(43): 3232-3240.

11. Cristi-Montero C, Steell L, Petermann F, Garrido-Mendez A, Diaz-Martinez X, Salas-Bravo C, et al. Joint effect of physical activity and sedentary behaviour on cardiovascular risk factors in Chilean adults. J Public Health (Oxford, England) 2017: Oct 17: 1-8.

12. Steell L, Garrido-Mendez A, Petermann F, Diaz-Martinez X, Martinez MA, Leiva AM, et al. Active commuting is associated with a lower risk of obesity, diabetes and metabolic syndrome in Chilean adults. J Public Health (Oxford, England) 2017: Jul 28: 1-9.

13. Garrido-Mendez A, Diaz X, Adela Martinez M, Maria Leiva A, Alvarez C, Ramirez Campillo $R$, et al. Association of active commuting with obesity: findings from the Chilean National Health Survey 2009-2010. Rev Med Chile 2017; 145(7): 837844

14. Celis-Morales CA, Lyall DM, Welsh P, Anderson J, Steell L, Guo $Y$, et al. Association between active commuting and incident cardiovascular disease, cancer, and mortality: prospective cohort study. Brit Med J 2017; 357: j1456.

15. Díaz-Martínez, X., Petermann, F., Leiva, A. M., Garrido-Méndez, A., Salas-Bravo, C., Martínez, M. A., \& Poblete-Valderrama, F. Association of physical inactivity with obesity, diabetes, hypertension and metabolic syndrome in the chilean population. Rev Med Chil 2018; 145 (5): 585-595.

16. Yannakoulia M, Ntanasi E, Anastasiou CA, Scarmeas N. Frailty and nutrition: From epidemiological and clinical evidence to potential mechanisms. Metabolism 2017; 68: 64-76.

17. Shlisky J, Bloom DE, Beaudreault AR, Tucker KL, Keller HH, Freund-Levi $Y$, et al. Nutritional Considerations for Healthy Aging and Reduction in Age-Related Chronic Disease. Adv Nutr 2017; 8(1): 17-26.

18. McPhee JS, French DP, Jackson D, Nazroo J, Pendleton N, Degens H. Physical activity in older age: perspectives for healthy ageing and frailty. Biogerontology 2016; 17(3): 567580 .

19. Vina J, Rodriguez-Manas L, Salvador-Pascual A, Jose TarazonaSantabalbina F, Carmen Gomez-Cabrera M. Exercise: the lifelong supplement for healthy ageing and slowing down the onset of frailty. J Physiol (London) 2016; 594(8): 1989-1999.

20. Bray NW, Smart RR, Jakobi IM, Jones GR. Exercise prescription to reverse frailty. App Physiol Nutr Metab 2016; 41(10): 11121116.

21. Hallal PC, Andersen LB, Bull FC, Guthold R, Haskell W, Ekelund U. Global physical activity levels: surveillance progress, 
pitfalls, and prospects. Lancet 2012; 380(9838): 247-257.

22. Dumith SC, Hallal PC, Reis RS, Kohl HW, 3rd. Worldwide prevalence of physical inactivity and its association with human development index in 76 countries. Prev Med 2011; 53(1-2): 24-28.

23. Garcia-Hermoso A, Saavedra JM, Ramirez-Velez R, Ekelund U, Del Pozo-Cruz B. Reallocating sedentary time to moderateto-vigorous physical activity but not to light-intensity physical activity is effective to reduce adiposity among youths: a systematic review and meta-analysis. Obes Rev 2017; 18(9): 1088-1095.

24. Ministry of Health of Chile, PUC. National Health Survey 2009-2010, Chile. Santiago, Chile 2010. [Avaible in: http:// web. minsal.cl/portal/url/item/bcb03d7bc28b64dfe 040010165012d23. pdf].

25. Cohen-Mansfield J, Shmotkin D, Goldberg S. Predictors of Longitudinal Changes in Older Adults' Physical Activity Engagement. J Aging Phys Act 2010; 18(2): 141-157.

26. World Health Organization. Obesity and overweight. 2014. [Available in: http://www.who.int/mediacentre/factsheets/ fs311/en/].

27. World Health Organization. Global Physical Activity Questionnaire: GPAQ version 2.0. 2009. http://www.who. int/ncds/surveillance/steps/resources/CPAQ_Analysis_Guide. pdf

28. Lee IM, Shiroma EJ, Lobelo F, Puska P, Blair SN, Katzmarzyk PT, et al. Effect of physical inactivity on major non-communicable diseases worldwide: an analysis of burden of disease and life expectancy. Lancet 2012; 380(9838): 219-229.

29. Bicalho PG, Hallal PC, Gazzinelli A, Knuth AG, VelasquezMelendez G. Adult physical activity levels and associated factors in rural communities of Minas Gerais State, Brazil. Rev Saude Publica 2010; 44(5): 884-893.

30. Hamrik, Z., Sigmundová, D., Kalman, M., Pavelka, J., Sigmund, E. Physical activity and sedentary behaviour in Czech adults: results from the GPAQ study. European journal of sport science 2014; 14(2): 193-198.

31. Ingram $D K$. Age-related decline in physical activity: generalization to nonhumans. Med Sci Sports Exerc. 2000; 32(9): 1623-1629.

32. Bauman AE, Reis RS, Sallis JF, Wells JC, Loos RJ, Martin BW. Correlates of physical activity: why are some people physically active and others not? Lancet. 2012; 380(9838): 258-271.

33. Thomaz PMD, Costa THMd, Silva EFd, Hallal PC. Factors associated with physical activity in adults, Brasilia, DF. Rev Saúde Pública. 2010; 44: 894-900.

34. Ximena Díaz, Alexis Garrido, María Adela Martínez, Ana María Leiva, Cristian Álvarez, Rodrigo Ramírez-Campillo, et al. F Correlates of physical inactivity: Findings from the Chilean National Health Survey 2009-2010. Rev Med Chile. 2017; 145(10): 1259-1267.

35. Henriksson $H$, Henriksson P, Tynelius $P$, et al. Muscular weakness in adolescence is associated with disability 30 years later: a population-based cohort study of 1.2 million men. Br J Sports Med Published Online First: 19 June 2018.

36. Aguilar-Farias N, Leppe Zamora J. Is a single question of the Global Physical Activity Questionnaire (GPAQ) valid for measuring sedentary behaviour in the Chilean population? J Sports Sci 2016: 1-6.

37. Celis-Morales CA, Perez-Bravo F, Ibañez L, Salas C, Bailey ME, Gill JM. Objective vs. self-reported physical activity and sedentary time: effects of measurement method on relationships with risk biomarkers. PLoSOne 2012; 7(5): e36345. 